\title{
Protective effects of hepatic stellate cells against cisplatin-induced apoptosis in human hepatoma $\mathbf{G 2}$ cells
}

\author{
LEI ZHANG $^{1,2^{*}}$, YI LI ${ }^{3 *}$, LIANG QIAO $^{4}$, YONGXUN ZHAO ${ }^{5}$, YUCAI WEI ${ }^{1,6}$ and YUMIN LI ${ }^{1,6}$ \\ ${ }^{1}$ Key Laboratory of Digestive System Tumors of Gansu Province, Lanzhou, Gansu 730030; \\ ${ }^{2}$ Department of Emergency Medicine, Sun Yat-sen Memorial Hospital of Sun Yat-sen University, \\ Guangzhou, Guangdong 510120; ${ }^{3}$ School of Basic Medical Science, Lanzhou University, Lanzhou, Gansu 730000, \\ P.R. China; ${ }^{4}$ Storr Liver Unit at the Westmead Millennium Institute, the University of Sydney at Westmead Hospital, \\ Westmead, NSW 2145, Australia; ${ }^{5}$ Department of Surgical Oncology, the First Hospital of Lanzhou University, \\ Lanzhou, Gansu 730000; ${ }^{6}$ The Second Hospital of Lanzhou University, Lanzhou, Gansu 730030, P.R. China
}

Received March 18, 2015; Accepted April 24, 2015

DOI: 10.3892/ijo.2015.3024

\begin{abstract}
The effects of hepatic stellate cells (HSCs) on tumorigenicity of HCC have been previously reported. However, the detailed mechanisms responsible for these effects remain unclear. In this study, we investigated the effects of HSCs on cisplatin-induced apoptosis in human hepatoma HepG2 cell lines. HepG2 cells were treated with cisplatin alone or co-cultured with LX-2 cells 3 days before incubation with cisplatin. Cisplatin causes apoptosis in HepG2 cells and LX-2 cells protect HepG2 cells from death. The protection of LX-2 cells against cisplatin-induced cytotoxicity in HepG2 cells appeared to be related to the inhibition of apoptosis, as determined by cytotoxicity assay and nuclear staining analysis. p53 and Bax mRNA levels were elevated, and cell cycle arrest was produced after cisplatin treatment. LX-2 cells suppressed this elevation of p53 and Bax as well as the cell cycle arrest induced by cisplatin, when compared with those of the treated cells with cisplatin alone. The LX-2 cells pretreatment inhibited the cisplatin-induced apoptosis, which was related with the incomplete blockage in p53 activation.
\end{abstract}

Correspondence to: Professor Yumin Li, Key Laboratory of Digestive System Tumors of Gansu Province, The Second Hospital of Lanzhou University, 82 Cuiyingmen, Lanzhou, Gansu 730030, P.R. China

E-mail: liym@lzu.edu.cn

*Contributed equally

Abbreviations: Bax, BCL2-associated X; BCA, bicinchoninic acid; Bcl-2, B-cell lymphoma 2; CCK-8, WST-8 Cell Counting Kit-8; ECM, extracellular matrix; FBS, fetal bovine serum; GAPDH, glyceraldehyde-3-phosphate dehydrogenase; HCC, hepatocellular carcinoma; HSCs, hepatic stellate cells; PBS, phosphate-buffered saline; PMSF, phenylmethanesulfonyl fluoride; PI, propidium iodide; SD, standard deviation

Key words: apoptosis, hepatic stellate cells, hepatocellular carcinoma
In summary, the results of our present study demonstrate that HSCs protect HepG2 cells against cisplatin-induced apoptosis and its protective effects occur via inhibiting the activation of p53, which is of critical importance for enhanced understanding of fundamental cancer biology.

\section{Introduction}

Hepatocellular carcinoma (HCC) is the sixth most frequently diagnosed cancer and the third most common leading cause of cancer-related death worldwide, and the burden of this devastating cancer is expected to increase in coming years $(1,2)$. The majority of patients are diagnosed at advanced stage and thus not in a position for curative treatments (3), which have very limited options, and the only systemic therapy currently approved is sorafenib, an oral tyrosine kinase inhibitor drug targeting multiple signalling pathways. The objective response rate (ORR), however, was poor (4). Cisplatin is a widely used anticancer agent and has a broad range of antitumor activity, which is likely a result of inhibition of replication by cisplatinDNA adducts and induction of apoptosis (5). HCC is considered a poor responder to chemotherapy and a standard systemic treatment does not exist for patients for whom sorafenib is unsuitable or unavailable. The reason could be the frequently observed development of multidrug tumor resistance, which is related to the high expression of the multidrug-resistance gene (6-9). The progression of cancer is no longer recognized as an independent event which only relates to the genetic mutation and uncontrollable growth of cancer cells. New therapeutic strategies are urgently needed, and these will most likely result from a better understanding of the cell biology of HCC.

The interaction between tumor cells and their microenvironment has been recognized to fundamentally affect tumor progression (10-12). Physiologically, the surrounding microenvironment constitutes an important barrier to cell transformation and notably modulate cell growth (13). Under cancer conditions, the tumor microenvironment experiences drastic changes including the recruitment and the activation of stromal cells and the remodeling of extracellular matrix 
(ECM), may influence the phenotype of tumor cells and provide a selective pressure for tumor initiation, progression and metastasis $(14,15)$.

The remodeling of tumor microenvironment is one of the hallmarks of HCC pathogenesis (16). The majority of HCC patients have an established background of chronic liver disease (17), and the presence of liver cirrhosis is the main risk factor for the development of HCC $(18,19)$. In addition, HCC is the leading cause of death among patients with cirrhosis (20). Hepatic stellate cells (HSCs), the versatile mesenchymal cells in the liver parenchyma, are vital to the liver's response to inflammation. Activation of HSCs is recognized as a central event in the development of hepatic fibrosis and subsequent, cirrhosis (21). In response to liver injury, quiescent HSCs become activated and convert into highly proliferative myofibroblast-like cells, which express inflammatory and fibrogenic mediators responsible for ECM accumulation within the microenvironment, and may affect proliferation, invasiveness and metastasis of tumor cells, and facilitates hepatic tumorigenesis (21-24). However, the detailed mechanisms responsible for these effects remain unclear.

Tumor mass is a balance between cell proliferation and death, and factors affecting this balance have a profound effect on tumor growth. Regulation of apoptosis in tumor cells remains poorly understood. Furthermore, few studies demonstrate the effects of HSCs on HCC from the chemotherapy-resistance perspective.

The aim of our study here was to examine if HSCs could provide a survival signal, or block a death signal, thereby protecting HCC cells from chemotherapy-induced apoptosis.

\section{Materials and methods}

Reagents. Cisplatin was purchased from Sigma-Aldrich (Shanghai) Trading Co., Ltd. (Shanghai, China). RNAiso Plus reagent, Primescript ${ }^{\mathrm{TM}}$ reverse transcription (RT) reagent kit and SYBR ${ }^{\circledR}$ Premix Ex Taq ${ }^{\mathrm{TM}}$ II were obtained from Takara Biotechnology Co. (Dalian, China). WST-8 Cell Counting Kit-8 (CCK-8), Hoechst 33258 staining kit, RIPA lysis buffer and bicinchoninic acid (BCA) were purchased from Beyotime Biotechnology (Haimen, China). Monoclonal antibody raised in rabbit against Bax (ab32503), Bcl-2 (ab136285) and monoclonal antibody raised in mouse against p53 (ab28), $\beta$-actin (ab8224) were obtained from Abcam Inc. (Cambridge, UK). Horseradish peroxidase (HRP)-conjugated goat anti-rabbit or anti-mouse $\operatorname{IgG}$ was purchased from Zhongshan Golden Bridge Biotech (Beijing, China).

Cell lines and cell culture. HepG2 human hepatoma cells (Cell Collection of the Chinese Academy of Sciences, Shanghai, China) and HSCs, LX-2 (kindly provided by Professor Hong Chen, Lanzhou University First Hospital, Lanzhou University, Lanzhou, China) were maintained by Dulbecco's modified Eagle's medium (DMEM) (HyClone Laboratories Inc., Logan, UT, USA) supplemented with heat-inactivated 10\% fetal bovine serum (FBS) (HyClone Laboratories Inc.), $100 \mathrm{U} / \mathrm{ml}$ penicillin and $100 \mu \mathrm{g} / \mathrm{ml}$ streptomycin (North China Pharmaceutical Co., Inc., Shijiazhuang, China) in a humidified atmosphere containing $5 \% \mathrm{CO}_{2}$ at $37^{\circ} \mathrm{C}$. The cells in mid-log phase were used in the experiments.

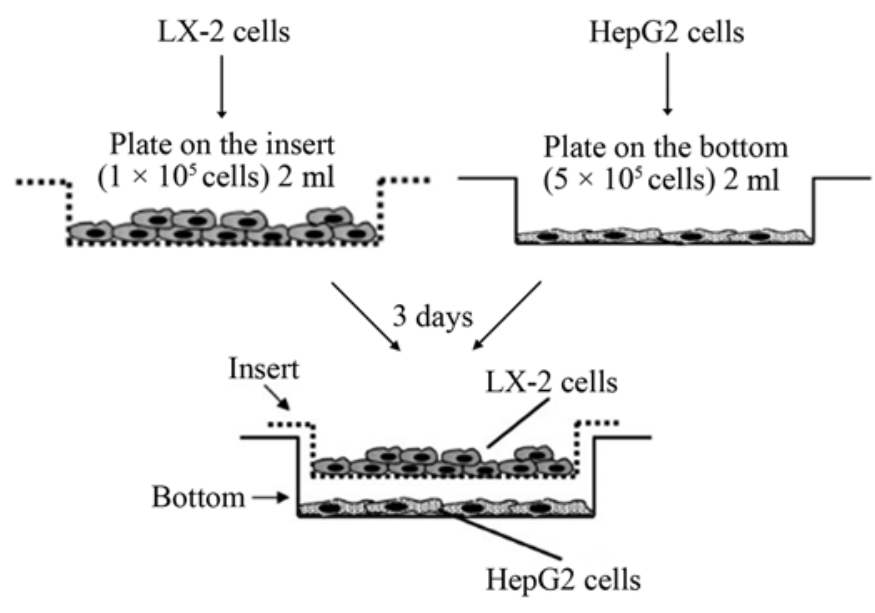

Figure 1. Scheme of the co-culture model. LX-2 cells were seeded on the insert plate at a density of $1 \times 10^{5}$ cells in $2 \mathrm{ml}$ of culture medium, and $5 \times 10^{5}$ HepG 2 cells were plated on the bottom in $2 \mathrm{ml}$ of culture medium. After 3 days incubation, HepG2 cells in the bottom were collected for all experiments.

The model used in the experiments described below is based on the co-culture of HepG2 cells with LX-2 cells. Details for the co-culture model are shown in Fig. 1. Cells were co-incubated using cell culture inserts of $1.0-\mathrm{mm}$ pore size (Merck Chemicals Co., Ltd, Shanghai, China) to separate both cell populations; this allows study of the effect of mediators from LX-2 cells on HepG2 cells, which resembles the physiological situation in the liver. The ratio of HepG2 cells to LX-2 cells was 5:1, similar to that of parenchymal: nonparenchymal cells in liver (25). HepG2 cells, after co-culturing for 3 days, were carefully removed from the 6 -well plates. They were washed twice with PBS, and the cells were removed using $0.25 \%$ trypsin and collected by centrifugation at $1000 \mathrm{rpm}$ for $5 \mathrm{~min}$. At this time all additions were made. In addition, the designation HepG2 refers to data found in HepG2 cells incubated with an empty insert; HepG2/1X-2 refers to results obtained in HepG2 cells after co-culture with the LX-2 cells.

Cytotoxicity assay. The viability of the cultured cells was analyzed using a CCK-8 colorimetric assay as described previously (26). In pilot experiments we tested cisplatin with varying concentrations to obtain an optimal concentration of cisplatin and chose a similar OD value to ensure the effect of cisplatin were comparable. The cells were seeded in 96-well flat bottom microtiter plates at a density of $5 \times 10^{3}$ cells per well, allowed to adhere in $5 \% \mathrm{CO}_{2}$ incubator at $37^{\circ} \mathrm{C}$, and appropriate concentrations of cisplatin ranging from 0.5 to $32.0 \mu \mathrm{g} / \mathrm{ml}$ (5) were then added. Control group and zero adjustment wells were also set. After incubation for $24-72 \mathrm{~h}, \mathrm{CCK}-8$ solution $(10 \mu \mathrm{l})$ was added to each well, the cells were incubated at $37^{\circ} \mathrm{C}$ for $1 \mathrm{~h}$ and then the absorbance at a test wavelength of $450 \mathrm{~nm}$ was measured using an automatic multiwell spectrophotometer (PowerWave $\mathrm{X}$, Bio-Tek Instruments Inc., Winooski, VT, USA). At least three independent experiments were performed.

Flow cytometry for cell cycle analysis. Cell cycle distribution was analyzed by flow cytometry. HepG2 cells were treated with cisplatin alone or pretreated with LX-2 cells for 3 days before incubation with cisplatin for $24 \mathrm{~h}$. Cells were harvested 

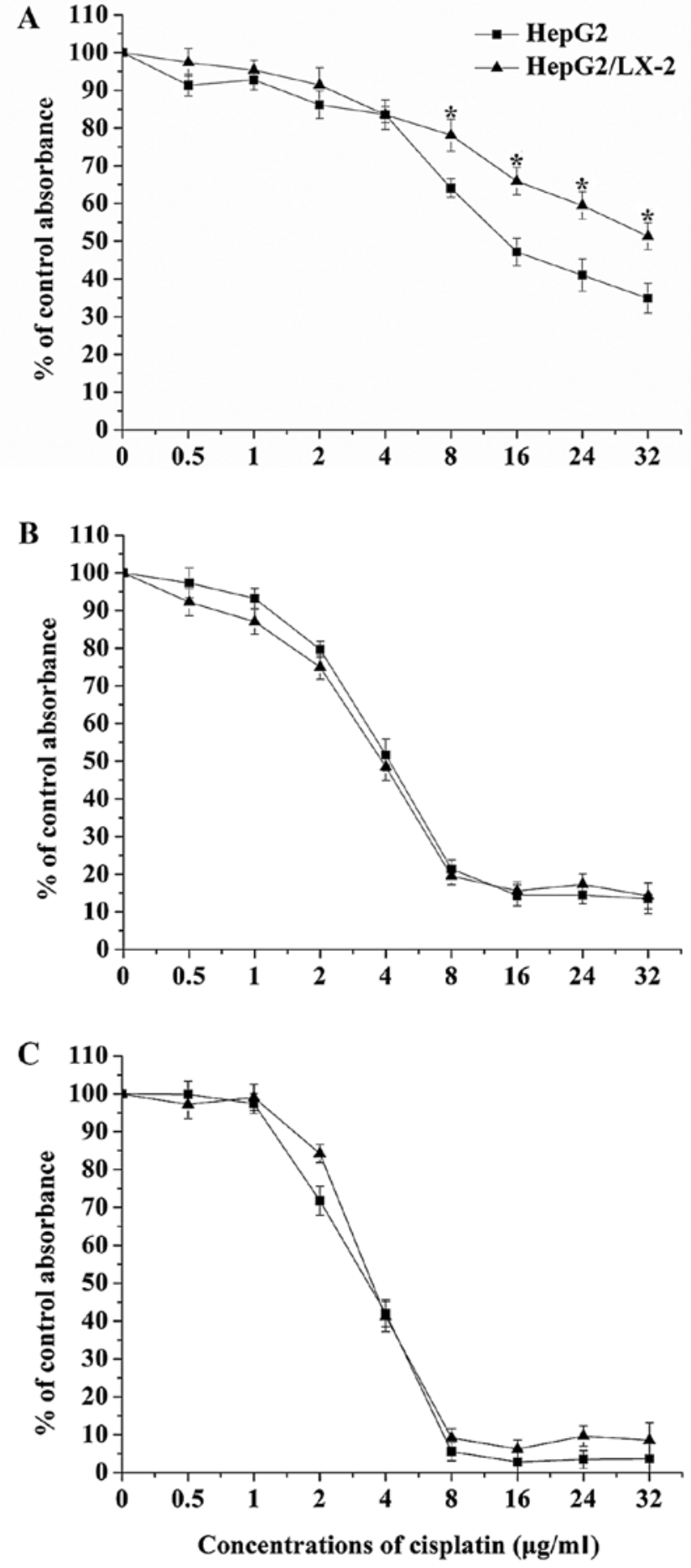

Figure 2. LX-2 cells suppress cisplatin-induced apoptosis in HepG2 cells. HepG2 cells were treated with cisplatin alone or pretreated with LX-2 cells for 3 days before incubation with cisplatin for 24 (A), 48 (B) and $72 \mathrm{~h}(\mathrm{C})$. The levels of cell survival were determined via CCK-8 assays and are expressed as percentages of cell growth relative to untreated controls. The values were expressed as means $\pm \mathrm{SD}$ of three independent experiments. ${ }^{*} \mathrm{P}<0.05$.

by trypsinization and washed with cold PBS, followed by centrifugation at $2000 \mathrm{rpm}$ for $10 \mathrm{~min}$. The pellet of cells was resuspended in $70 \%$ cold ethanol and stored at $4^{\circ} \mathrm{C}$ overnight. Cells were washed twice with PBS. The pellet was resuspended in PBS containing $20 \mu \mathrm{g} / \mathrm{ml}$ RNase A, $3.4 \mathrm{mmol} / \mathrm{l}$ sodium citration and $1 \%$ Triton X-100 in the dark, incubated at room temperature for $30 \mathrm{~min}$, stained with PI (50 $\mu \mathrm{g} / \mathrm{ml})$, and
Table I. Primer sequences used to detect Bax, Bcl-2 and p53 for qPCR.

\begin{tabular}{llc}
\hline Gene & \multicolumn{1}{c}{ Primer sequence } & $\begin{array}{c}\text { Product size } \\
\text { (bp) }\end{array}$ \\
\hline Bax & F: 5'-ATCCAAGACCAGGGTGGTT-3' & 125 \\
& R: 5'-ATCTGGAAGAAGATGGGCTG-3' & \\
Bcl-2 & F: 5'-GGCCTCTGTTTGATTTCTCC-3' & 116 \\
& R: 5'-AGTGAAGTCAACATGCCTGC-3' & \\
p53 & F: 5'-TACATCTGGCCTTGAAACCA-3' & 119 \\
& R: 5'-TTTCTACAGTTGGGCAGCTG-3' & \\
GAPDH & F: 5'-ATCATCCCTGCCTCTACTGG-3' & 122 \\
& R: 5'-GTGTCAGTGGTGGACCTGAC-3' & \\
\hline
\end{tabular}

$F$, forward; $R$, reverse.

analyzed by EPICS XL flow cytometry (Beckman Coulter, Brea, CA, USA). Each assay was performed in triplicate.

Morphologic observation. HepG2 cells were equally seeded in 24-well plate (Costar 3524, Corning Inc., Corning, NY, USA) and then treated with cisplatin alone or pretreated with LX-2 cells for 3 days before incubation with cisplatin for $24 \mathrm{~h}$. The cells were washed gently three times with PBS. The morphology of HepG2 and HepG2/1X-2 cells was observed under an inverted phase contrast microscope (Olympus, Tokyo, Japan).

Nuclear staining analysis by Hoechst 33258. Nuclear morphology of apoptotic cells was also examined after nuclear staining using Hoechst 33258 staining kit. Briefly, after the drug treatment, the cells were fixed with $4 \%$ paraformaldehyde for $30 \mathrm{~min}$ and washed three times with PBS. Then cells were stained with $10 \mathrm{mg} / \mathrm{l}$ Hoechst 33258 in the dark at room temperature for $10 \mathrm{~min}$. Morphologic changes in apoptotic nuclei were observed under fluorescence microscope. The cells with condensed chromatin and shrunken nuclei were classified as apoptotic cells. Apoptosis was assessed by counting the number of apoptotic cells in five random fields per slide. At least three slides were counted.

Quantitative real-time reverse transcription-polymerase chain reaction ( $q R T-P C R)$ assay. Total RNA was isolated from the cultured cells using RNAiso Plus reagent following the recommendation of the manufacturer, then reverse transcribed into cDNA using the Primescript reverse transcription (RT) Master Mix according to manufacturer's instructions. Reverse transcription reaction was performed at $37^{\circ} \mathrm{C}$ for $15 \mathrm{~min}$ followed by $85^{\circ} \mathrm{C}$ for $5 \mathrm{sec}$. qRT-PCR amplifications were performed using a standard protocol and undertaken with Applied Biosystems 7500/7500 Fast Real-time PCR Software (Applied Biosystems, Foster, CA, USA) using the SYBR Premix Ex Taq II. Reactions were carried out in a $20-\mu l$ reaction volume. The thermal cycle conditions were as follows: $95^{\circ} \mathrm{C}$ for $30 \mathrm{sec}$, followed by 40 cycles of $95^{\circ} \mathrm{C}$ for $5 \mathrm{sec}$ and $60^{\circ} \mathrm{C}$ for $34 \mathrm{sec}$. The primers used in the PCR reactions are described in Table I. The level of gene expression 


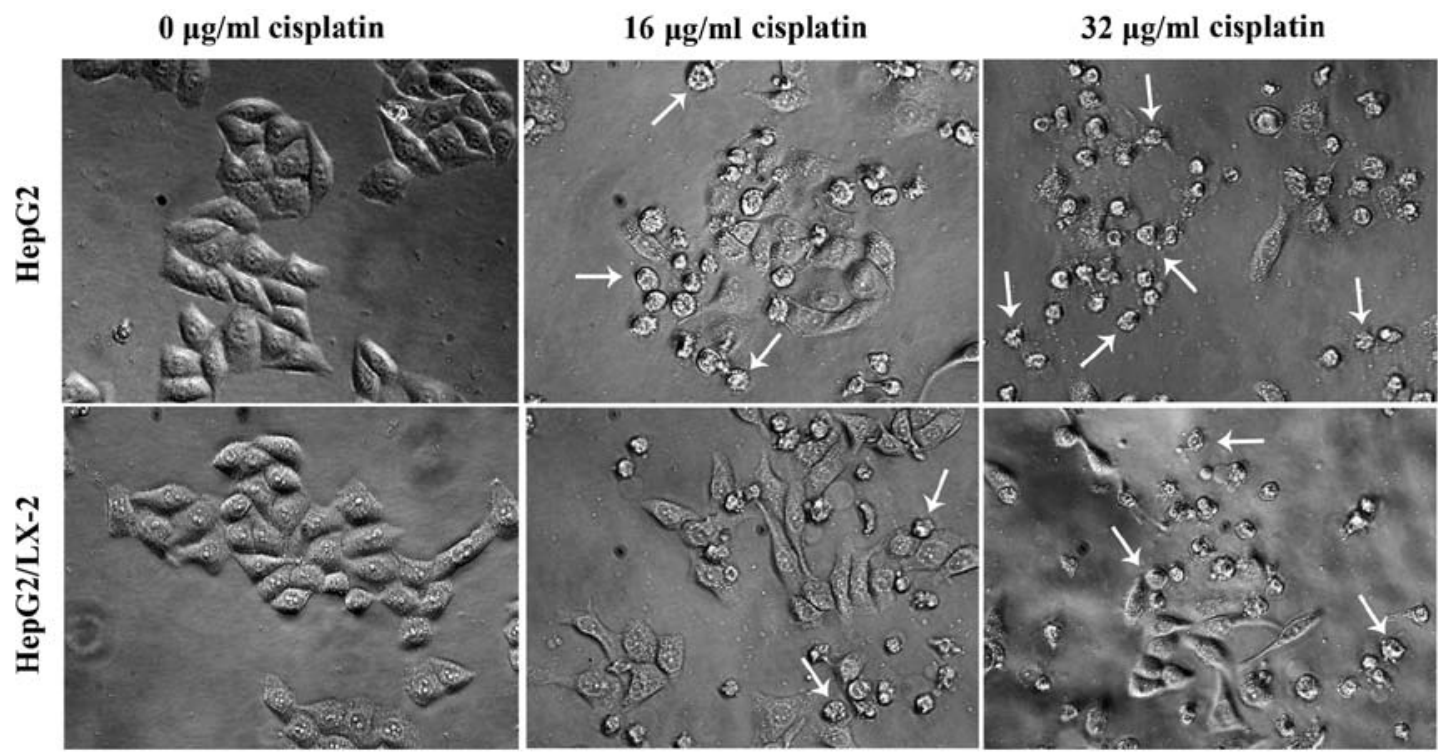

Figure 3. Morphology of HepG2 and HepG2/1X-2 cells treated with cisplatin. Morphology was visualized and photographed under a light microscope. White arrowheads in the pictures indicate the nuclei of apoptotic cells. (magnification, $\mathrm{x} 400$ ).

was normalized with GAPDH. All primers were synthesized by Takara Biotechnology Co. Amplification was performed at least thrice in three independent experiments. Data were analyzed according to the comparative $\mathrm{Ct}$ method.

Western blotting. Western blots were performed and analyzed as previously described (27). Treated cells were washed with ice-cold PBS and then lysed using RIPA lysis buffer supplemented with $1 \mathrm{mM}$ PMSF. After centrifugation at 12,000 rpm for $15 \mathrm{~min}$ at $4^{\circ} \mathrm{C}$, the supernatant was collected and the protein concentration was measured by the BCA protein assay. Forty micrograms of protein per sample were loaded on SDS-polyacrylamide gel $(10 \%)$ and transferred to polyvinylidene fluoride (PVDF) membranes. The blots were blocked with 5\% skimmed milk in Tris-buffered saline containing $0.1 \%$ Tween-20 (TBST) for $1 \mathrm{~h}$ at room temperature and then incubated at $4^{\circ} \mathrm{C}$ overnight with primary antibodies against Bax (1:1000), Bcl-2 (1:800) and p53 (1:1000). $\beta$-actin (1:4000) was used as a loading control. After being washed in TBST three times, the membranes were incubated with horseradish peroxidase-conjugated secondary antibody $(1: 10,000)$ for $1 \mathrm{~h}$ at room temperature. The bands were visualized with the SuperSignal West Pico Chemiluminescent Substrate (Thermo Fisher Scientific, Inc., Rockford, IL, USA) and imaged using a VersaDoc Imaging System (Bio-Rad Laboratories Co., Ltd. Hercules, CA, USA). Densitometric analysis was performed using Quantity One Software v4.62 (Bio-Rad Laboratories Co.) and the results were presented as the mean of three independent experiments.

Statistical analysis. Values are shown as mean \pm standard deviation (SD) of at least three independently performed experiments to avoid possible variation. All statistical analysis were carried out using SPSS 19.0 (IBM, Armonk, NY, USA). Statistical significance was determined with Student's t-test and one-way ANOVA followed by the Bonferroni correction. A P-value of $<0.05$ was considered statistically significant.

\section{Results}

LX-2 cells protect cisplatin-induced cytotoxicity in HepG2 cells. In order to evaluate the effects of LX-2 cells on cisplatin-induced cytotoxicity in HepG2 cells, HepG2 cells were pretreated with LX-2 cells for 3 days and subsequently treated with increasing concentrations cisplatin. As shown in Fig. 2, the results showed that cisplatin inhibited the proliferation of HepG2 and HepG2/1X-2 cells in a time- and concentrationdependent manner. However, LX-2 cells protected HepG2 cells from cell death induced by cisplatin, significantly in the presence of 8.0-32.0 $\mu \mathrm{g} / \mathrm{ml}$ cisplatin for $24 \mathrm{~h}$. $\mathrm{IC}_{50}$ defined as the drug concentrations resulting in $50 \%$ loss of cell viability relative to the untreated cells was determined for HepG 2 and HepG2/1X-2 cells from Fig. 2 (Table II). The $\mathrm{IC}_{50}$ doses for cisplatin in HepG2 and HepG2/1X-2 cells were determined to be $16.09 \pm 1.52 \mu \mathrm{g} / \mathrm{ml}$ and $38.23 \pm 3.65 \mu \mathrm{g} / \mathrm{ml}$, respectively. Thus, cisplatin was more than 2.4 times more potent in HepG2 cells than in HepG2/1X-2 cells. Therefore, based on above results, $16.0 \mu \mathrm{g} / \mathrm{ml}$ (low concentration) and $32.0 \mu \mathrm{g} / \mathrm{ml}$ (high concentration) of cisplatin at $24 \mathrm{~h}$ were used to stand for appropriate and high doses, respectively, in the following experiments.

LX-2 cells suppress cisplatin-induced apoptosis in HepG2 cells. To further evaluate the effects of LX-2 cells on cisplatininduced apoptosis in HepG2 cells, HepG2 cells were pretreated with LX-2 cells for 3 days, and then incubated them with cisplatin high $(32 \mu \mathrm{g} / \mathrm{ml})$ and low $(16 \mu \mathrm{g} / \mathrm{ml})$ concentrations. As shown in Fig. 3, after exposure to cisplatin for $24 \mathrm{~h}$, many of the cells exhibited cytoplasmic shrinkage and either detached from each other or floated in the medium. At high dose, there was a significant increase in the percentage of rounded cells with progressive nuclear shrinkage. The cells eventually became detached from the culture dish, and cytoplasmic vacuolation and cellular fragmentation were clearly seen. However, LX-2 cell pretreatment before cisplatin exposure to 
A

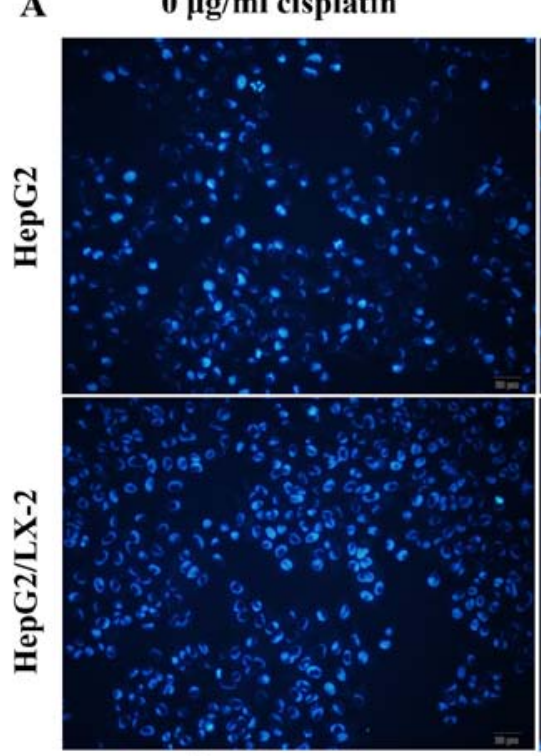

$16 \mu \mathrm{g} / \mathrm{ml}$ cisplatin

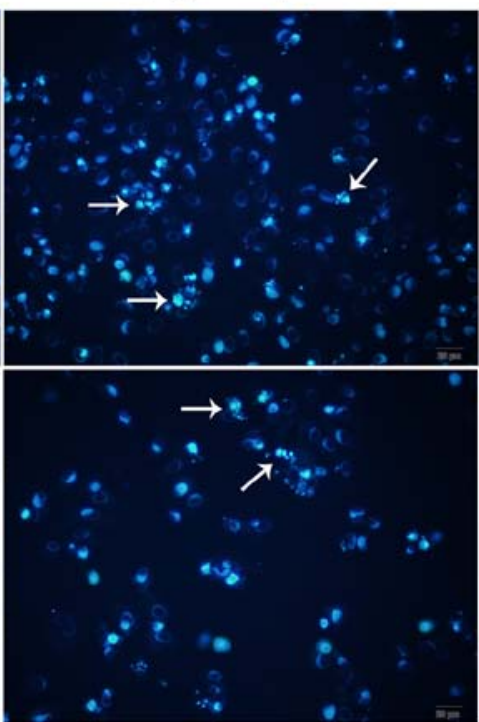

$32 \mu \mathrm{g} / \mathrm{ml}$ cisplatin

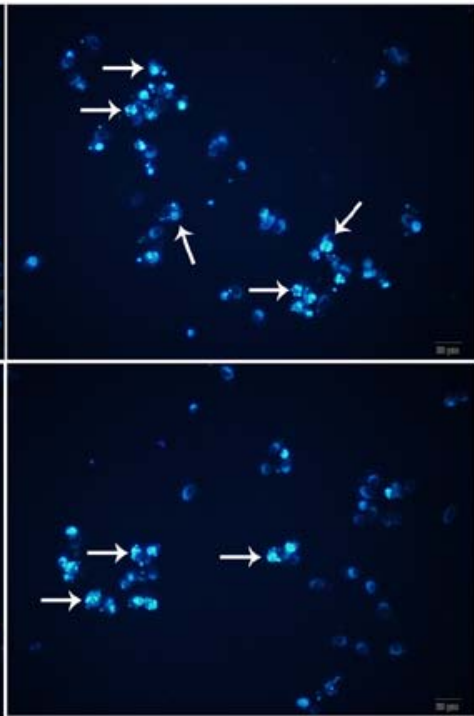

B

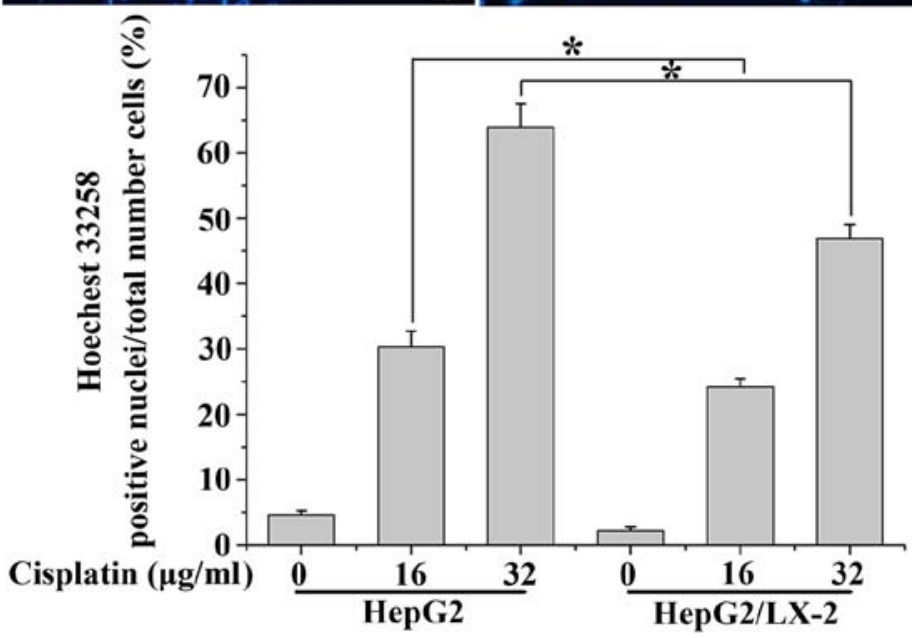

Figure 4. LX-2 cells suppress cisplatin-induced apoptosis in HepG2 cells. (A) Morphological changes associated with apoptosis were examined by Hoechst 33258 staining. (B) The apoptotic index was determined by (number of positively stained cells/total number of cells) x100\%. White arrowheads in the images indicate the nuclei of apoptotic cells. (magnification, $\mathrm{x} 200$ ). ${ }^{*} \mathrm{P}<0.05$.

Table II. Cisplatin cytotoxicity in HepG2 and HepG2/lX-2 cells.

\begin{tabular}{lccc}
\hline Cisplatin & & HepG2 & HepG2/lX-2 \\
\hline $24 \mathrm{~h}$ & $\mathrm{IC}_{50}$ & $16.09 \pm 1.52$ & $38.23 \pm 3.65^{\mathrm{a}}$ \\
\hline
\end{tabular}

The values are expressed as means \pm SD of three independent experiments. ${ }^{\mathrm{P}}<0.01$, significant compared between the HepG2 and HepG2/1X-2 cells.

HepG2 cells suppressed the morphological characteristics of apoptosis induced by cisplatin, and increased the number of surviving cells.

These results were further confirmed by a morphological analysis using nuclear staining analysis by Hoechst 33258 . The Hoechst fluorochrome was able to diffuse through intact membranes of cells and stain DNA. As shown in Fig. 4A, nuclei morphological change was observed by Hoechst 33258 staining, which illustrated that the control cells exhibited uniformly dispersed chromatin, normal organelle and intact cell membrane; exposed to cisplatin for $24 \mathrm{~h}$, cells featured typical characteristics of apoptosis, including the condensation of chromatin, the shrinkage of nuclei and the appearance of a few apoptotic bodies and $\sim 30.4 \%$ underwent apoptosis with cisplatin at the concentration $16.0 \mu \mathrm{g} / \mathrm{ml}, 63.7 \%$ with cisplatin at the concentration $32.0 \mu \mathrm{g} / \mathrm{ml}$; however, $\mathrm{LX}-2$ cells protected HepG2 cells from cell death induced by cisplatin, when compared with those of the treated cells with cisplatin alone ( $24.2 \%$ underwent apoptosis with cisplatin at the concentration $16.0 \mu \mathrm{g} / \mathrm{ml}, 46.9 \%$ with cisplatin at the concentration $32.0 \mu \mathrm{g} / \mathrm{ml}$ ) (Fig. 4B).

$L X-2$ cells reduce the Bax:Bcl-2 ratio in cisplatin-treated HepG2 cells. It is thought that the ratio of the pro-apoptotic protein Bax and the anti-apoptotic protein Bcl-2 plays a crucial role in the control of the intrinsic pathway of apoptosis. We therefore reasoned that the cell death inhibited by LX-2 cells in HepG2 cells treated with cisplatin might be due to changes in this ratio. The expression of Bcl-2 and Bax was probed by qRT-PCR and western blot analysis. As shown in Fig. 5A, 

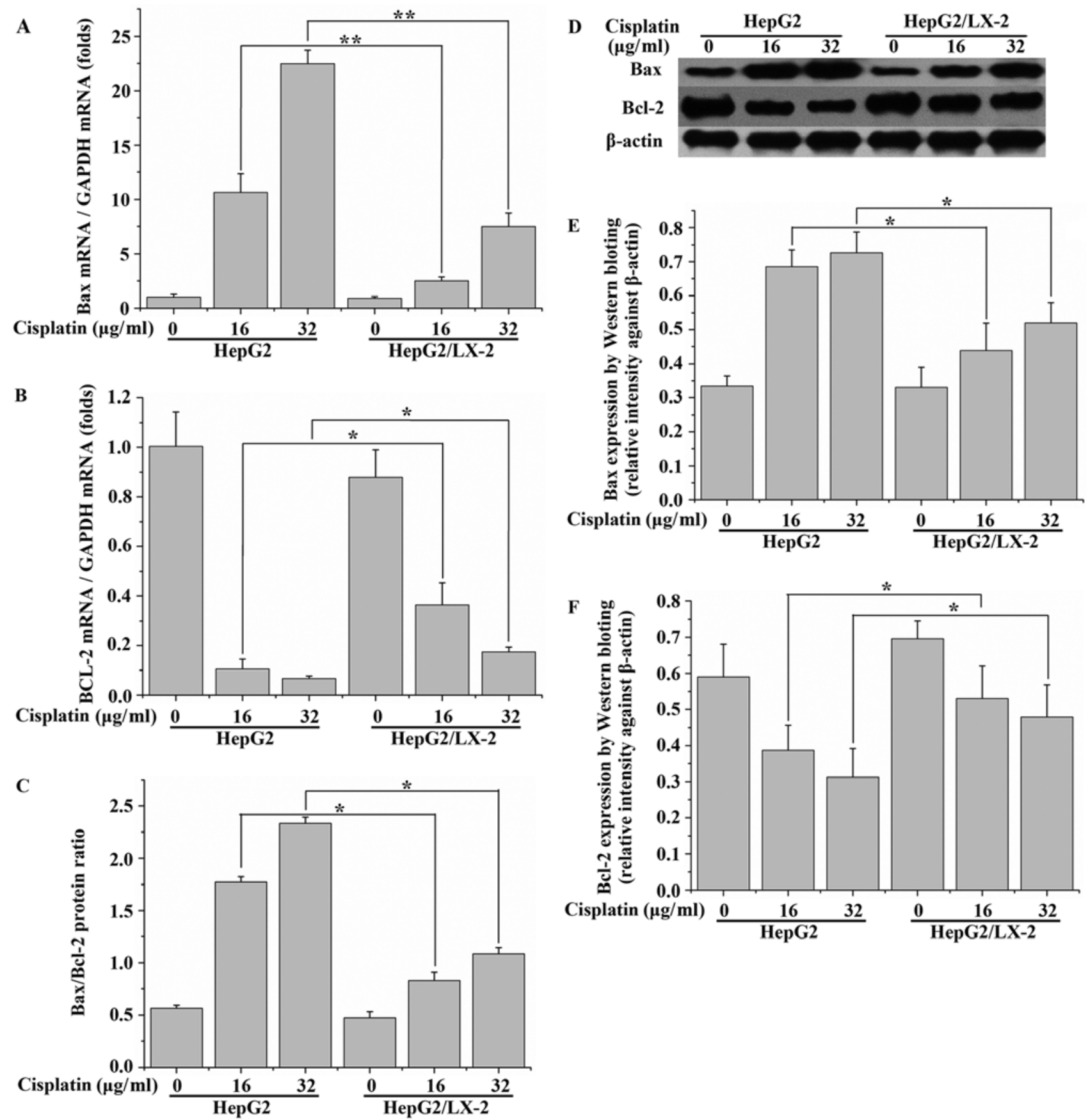

Figure 5. LX-2 cells suppress the expression of Bax and Bcl-2 in cisplatin-treated HepG2 cells. The expression of Bax (A), and Bcl-2 (B) mRNA were detected by qRT-PCR. (C) Ratio of Bax/Bcl-2 protein level. (D) The expression level of Bax and Bcl-2 were detected by western blot analysis, and (E and F) the results were quantitatively analyzed by densitometry. ${ }^{*} \mathrm{P}<0.05,{ }^{* *} \mathrm{P}<0.01$.

cisplatin upregulated the Bax mRNA expression in HepG2 cells in a dose-dependent manner. Following $24 \mathrm{~h}$ of treatment with 16.0 and $32.0 \mu \mathrm{g} / \mathrm{ml}$ cisplatin, the Bax mRNA expression level increased nearly 10 - and 20 -fold, respectively, however, pretreatment with LX-2 for 3 days induced a marked reduction in Bax mRNA expression. The results showed a reverse trend to that in Bcl-2 mRNA expression (Fig. 5B). These results were consistent with those obtained from western blot analysis by use of anti-Bax and anti-Bcl-2 antibodies (Fig. 5D-F). Treatment with $32.0 \mu \mathrm{g} / \mathrm{ml}$ cisplatin enhanced the ratio of Bax to Bcl-2 approximately 4.1 -fold, as compared with that for the untreated cells. However, when the cells were cultured with $32.0 \mu \mathrm{g} / \mathrm{ml}$ cisplatin together with LX-2 cells, this ratio was decreased to $\sim 55 \%$ of that for the cisplatin-treated cells (Fig. 5C). These results further demonstrated that LX-2 cells had the potential to suppress cisplatin-induced apoptosis in HepG2 cells.

LX-2 cells suppress the expression of $p 53$ and cell cycle arrest in cisplatin-treated Hep 2 cells. Cisplatin induces p53 activation in HepG2 cells. p53 level was elevated at $24 \mathrm{~h}$ after treated with cisplatin compared with control cells (Fig. 6C). LX-2 cells abolished this elevation of $\mathrm{p} 53$ induced by cisplatin. This suggests that LX-2 cells may play a critical role in cell-induced 

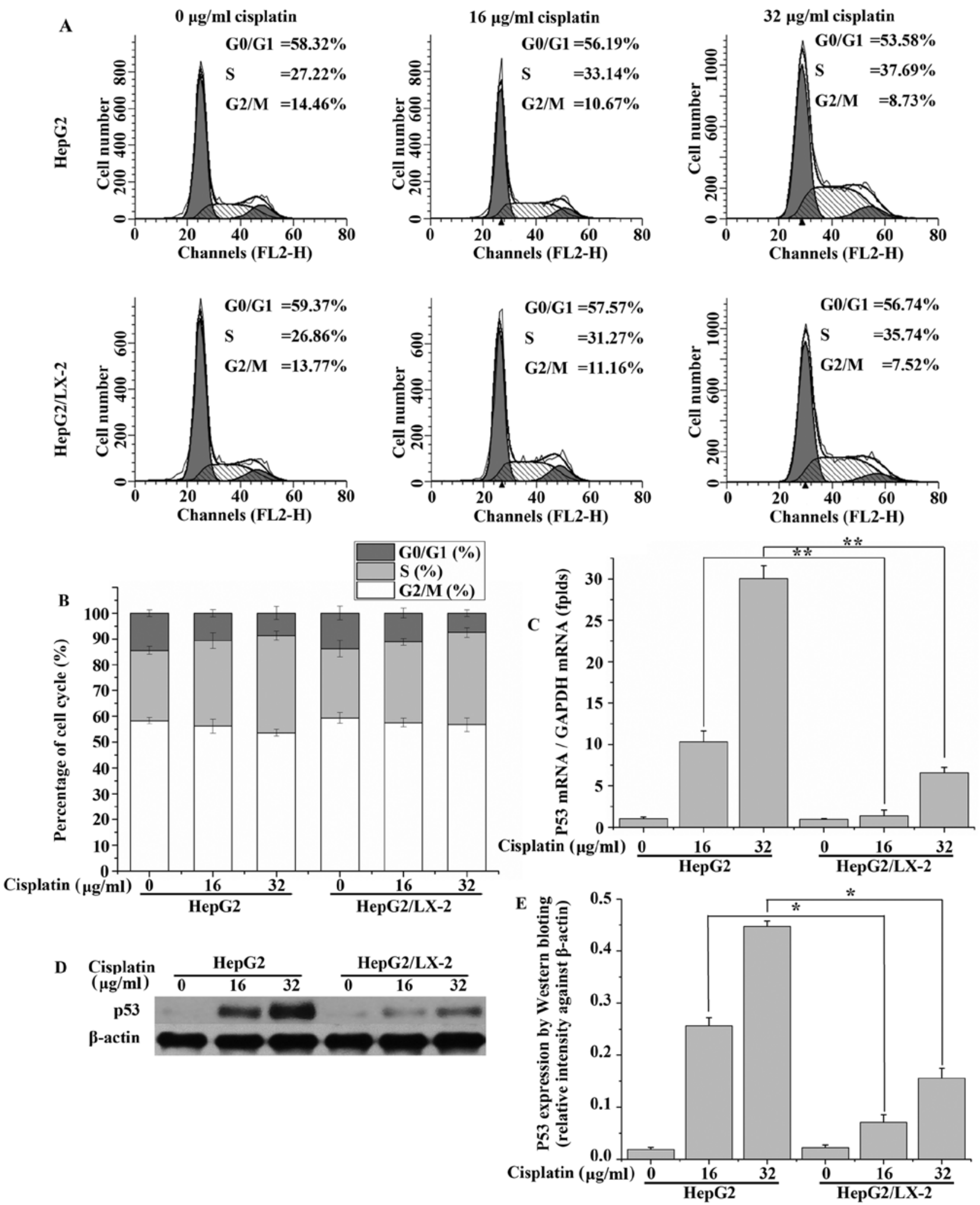

Figure 6. LX-2 cells suppress the expression of p53 and cell cycle arrest in cisplatin-treated HepG2 cells. (A) Flow cytometry DNA histographs of cells. (B) Percentage of cells in different stages of cell cycle were quantified. (C) The expression of p53 mRNA were detected by qRT-PCR. (D) The expression level of p53 was detected by western blot analysis, and the results (E) was quantitatively analyzed by densitometry. The values were expressed as means \pm SD of three independent experiments. ${ }^{*} \mathrm{P}<0.05,{ }^{* *} \mathrm{P}<0.01$.

resistance to the effects of anticancer drugs which upregulate p53. The same trend was seen in the protein expression levels of p53 (Fig. 6D-E). Cell cycle analysis showed that cisplatin caused G0/G1-S cell cycle arrest. The cell population in the
S phase increased in cells exposed to $32.0 \mu \mathrm{g} / \mathrm{ml}$ cisplatin for $24 \mathrm{~h}(37.69 \%)$ compared to cells without cisplatin treatment (27.22\%). LX-2 cells slightly suppress this increase induced by cisplatin (Fig. 6A and B). 


\section{Discussion}

The results of our present study demonstrate that LX-2 cells protect HepG2 cells against cisplatin-induced cytotoxicity and its protective effects occur via the anti-apoptosis pathway in HepG 2 cells. Flow cytometry showed that cisplatin arrested HepG2 cells in G0/G1-S phase of cell cycle, whereas LX-2 cells retarded the progression of cell cycle (Fig. 3), which may be one of the mechanisms underlying the protective effect of LX-2 cells on HepG2 cells.

The role of the microenvironment during the initiation and progression of carcinogenesis is now realized to be of critical importance, both for enhanced understanding of fundamental cancer biology, as well as exploiting this source of relatively new knowledge for improved molecular diagnostics and therapeutics $(28,29)$. The tumor microenvironment, developed from Paget's 'seed and soil' theory (30), which is a changing concept that defines the behavior of cancer not only by the tumor cells alone, but also by the surrounding microenvironment that the tumor cells need for survival (29). It has long been established that patients with chronic liver disease, and particularly those with cirrhosis, have an increased risk of developing hepatocellular carcinoma (19). Although the importance of the association of HCC with cirrhosis is still obscure, such an association provides a means to identify patients at high risk for HCC. The cross-talk between HCC cells and the surrounding tumor microenvironment is believed to play a pivotal role in modulating the biological behaviour of the tumor, likely affecting a different clinical outcome. Cisplatin is a widely used anticancer agent and has a broad range of antitumor activity (31). However, its curative effect is limited due to chemoresistance of $\mathrm{HCC}$, which remains a major clinical obstacle $(32,33)$. Thus, it is urgent to advance our understanding of mechanisms of chemoresistance and improve the efficacy of the current treatment strategies.

It has been demonstrated that various stroma cell types are recruited to neoplasms, where they substantially promote the proliferation, invasiveness and metastatic potential of cancer cells. HSCs belong to one of the most important stroma cell types in the liver tumor environment. Previous study demonstrated that activated HSCs promoted tumorigenicity of $\mathrm{HCC}$, stimulating proliferation and migration of three different human HCC cell lines in vitro and promote growth and invasiveness of human HCC cells in nude mice (22). However, the detailed molecular mechanisms underlying the chemopreventive effects of HSCs remained unclear. In this study, a novel activity of HSCs was identified in HepG2 cells, namely the protective effects from chemotherapy-induced apoptosis. In line with our data, we demonstrate the effects of HSCs on HCC from the chemotherapy-resistance perspective, which shows potential as a new cancer treatment modality.

In this study, cisplatin induced apoptosis of HepG2 cells in a time- and dose-dependent manner (Fig. 2). Consistent with the ability of cisplatin to kill HepG2 cells via apoptotic processes, cisplatin upregulated expression of the proapoptosis gene Bax, in a dose-dependent manner, whereas LX-2 cells inhibited this process (Fig. 5A), indicating that LX-2 cells were likely to protect HepG2 cells from death via restraining the apoptosis pathway. Apoptosis is the process of cell death characterized by cell shrinkage, nuclear condensation, DNA fragmentation, expression of apoptosis-related genes, and activation of the caspase cascade. The pro-apoptotic factor Bax resides in the cytosol, in contrast, the anti-apoptotic Bcl-2 is localized in the outer mitochondrial membrane, and this protein inhibits the release of cytochrome c. Translocation of Bax to the mitochondrial membrane might lead to a loss of mitochondrial membrane potential and an increase in mitochondrial permeability. Cisplatin downregulated expression of the anti-apoptosis gene $\mathrm{Bcl}-2$, in a dose-dependent manner, whereas LX-2 cells inhibited this change (Fig. 5B). In this study, abundant cytoplasmic vacuoles were observed in cisplatin-treated HepG2 cells under an inverted phase contrast microscope (Fig. 3). When LX-2 cells were applied, the number of apoptotic cells decreased. Hoechst 33258 staining demonstrated independent evidence supporting the conclusion that LX-2 cells has an antiapoptotic effect on HepG2 cells induced by cisplatin (Fig. 4). The above-mentioned apoptotic features indicated that LX-2 cells have anti-apoptotic effects on HepG2 cells.

LX-2 cells also suppress the G1/G0-S cell cycle arrest induced by cisplatin, most probably due to the decrease in p53. p53 is implicated in DNA repair, apoptosis and cell cycle control (34). Hepatoblastoma derived HepG2 cells, which have wild-type p53 (35), were more sensitive to cisplatin, suggesting that wild-type p53 might be one of the factors that modulates the sensitivity to cisplatin (35). In the current study, we found that cisplatin induces activation of p53. LX-2 cells decrease p53 levels and suppress cell death.

In summary, the present study further supports the impact of stromal activated HSCs on HCC progression in vitro. These results demonstrate that HSCs partially protect HepG2 cells against cisplatin-induced apoptosis and its protective effects occur via decreasing the level of p53. LX-2 cells may therefore play an important role in cell-induced resistance to the effects of anticancer drugs by modulating the cellular levels of p53. In further study, the precise molecular mechanism of the protective effect by LX-2 cells against cisplatin-induced apoptosis should be elucidated.

\section{Acknowledgements}

We wish to thank Dr Hongwen Zhu and Dr Yang Zhao (Lanzhou University Second Hospital) for many helpful suggestions and advice. This work was supported by the National Natural Science Foundation of China (Grant ID: 31270532) and the Fundamental Research Funds of the Central Universities, China (Grant ID: lzujbky-2013-m04).

\section{References}

1. Jemal A, Bray F, Center MM, Ferlay J, Ward E and Forman D: Global cancer statistics. CA Cancer J Clin 61: 69-90, 2011.

2. Yazici C, Niemeyer DJ, Iannitti DA and Russo MW: Hepatocellular carcinoma and cholangiocarcinoma: An update. Expert Rev Gastroenterol Hepatol 8: 63-82, 2014.

3. Patrikidou A, Sinapi I, Regnault H, Fayard F, Bouattour M, Fartoux L, Faivre S, Malka D, Ducreux M and Boige V: Gemcitabine and oxaliplatin chemotherapy for advanced hepatocellular carcinoma after failure of anti-angiogenic therapies. Invest New Drugs 32: 1028-1035, 2014.

4. Llovet JM, Ricci S, Mazzaferro V, Hilgard P, Gane E, Blanc JF, de Oliveira AC, Santoro A, Raoul JL, Forner A, et al: SHARP Investigators Study Group: Sorafenib in advanced hepatocellular carcinoma. N Engl J Med 359: 378-390, 2008. 
5. Qin LF and Ng IO: Induction of apoptosis by cisplatin and its effect on cell cycle-related proteins and cell cycle changes in hepatoma cells. Cancer Lett 175: 27-38, 2002.

6. Frenzel A, Grespi F, Chmelewskij W and Villunger A: Bcl2 family proteins in carcinogenesis and the treatment of cancer. Apoptosis 14: 584-596, 2009.

7. Wei W, Chua MS, Grepper S and So SK: Blockade of Wnt-1 signaling leads to anti-tumor effects in hepatocellular carcinoma cells. Mol Cancer 8: 76, 2009.

8. Venkatesan B, Prabhu SD, Venkatachalam K, Mummidi S, Valente AJ, Clark RA, Delafontaine P and Chandrasekar B: WNT1-inducible signaling pathway protein-1 activates diverse cell survival pathways and blocks doxorubicin-induced cardiomyocyte death. Cell Signal 22: 809-820, 2010

9. Germano D and Daniele B: Systemic therapy of hepatocellular carcinoma: Current status and future perspectives. World J Gastroenterol 20: 3087-3099, 2014.

10. Coulouarn C, Corlu A, Glaise D, Guénon I, Thorgeirsson SS and Clément B: Hepatocyte-stellate cell cross-talk in the liver engenders a permissive inflammatory microenvironment that drives progression in hepatocellular carcinoma. Cancer Res 72 : 2533-2542, 2012.

11. Hanahan D and Weinberg RA: Hallmarks of cancer: The next generation. Cell 144: 646-674, 2011.

12. Trimboli AJ, Cantemir-Stone CZ, Li F, Wallace JA, Merchant A, Creasap N, Thompson JC, Caserta E, Wang H, Chong JL, et al: Pten in stromal fibroblasts suppresses mammary epithelial tumours. Nature 461: 1084-1091, 2009.

13. Nelson CM and Bissell MJ: Of extracellular matrix, scaffolds, and signaling: Tissue architecture regulates development, homeostasis, and cancer. Annu Rev Cell Dev Biol 22: 287-309, 2006.

14. Joyce JA and Pollard JW: Microenvironmental regulation of metastasis. Nat Rev Cancer 9: 239-252, 2009.

15. Polyak K, Haviv I and Campbell IG: Co-evolution of tumor cells and their microenvironment. Trends Genet 25: 30-38, 2009.

16. Hao H, Liu M, Wu P, Cai L, Tang K, Yi P, Li Y, Chen Y and Ye D: Lipoxin A4 and its analog suppress hepatocellular carcinoma via remodeling tumor microenvironment. Cancer Lett 309: 85-94, 2011.

17. Sherman M: Hepatocellular carcinoma: Epidemiology, surveillance, and diagnosis. Semin Liver Dis 30: 3-16, 2010.

18. Farazi PA and DePinho RA: Hepatocellular carcinoma pathogenesis: From genes to environment. Nat Rev Cancer 6: 674-687, 2006.

19. Bruix J and Sherman M; American Association for the Study of Liver Diseases: Management of hepatocellular carcinoma: An update. Hepatology 53: 1020-1022, 2011.

20. Alazawi W, Cunningham M, Dearden J and Foster GR: Systematic review: Outcome of compensated cirrhosis due to chronic hepatitis C infection. Aliment Pharmacol Ther 32: 344-355, 2010.
21. Hernandez-Gea V and Friedman SL: Pathogenesis of liver fibrosis. Annu Rev Pathol 6: 425-456, 2011.

22. Amann T, Bataille F, Spruss T, Mühlbauer M, Gäbele E, Schölmerich J, Kiefer P, Bosserhoff AK and Hellerbrand C: Activated hepatic stellate cells promote tumorigenicity of hepatocellular carcinoma. Cancer Sci 100: 646-653, 2009.

23. Wang BB, Cheng JY, Gao HH, Zhang Y, Chen ZN and Bian H: Hepatic stellate cells in inflammation-fibrosis-carcinoma axis. Anat Rec (Hoboken) 293: 1492-1496, 2010.

24. Jia YL, Shi L, Zhou JN, Fu CJ, Chen L, Yuan HF, Wang YF, Yan XL, Xu YC,Zeng Q, et al: Epimorphin promotes human hepatocellular carcinoma invasion and metastasis through activation of focal adhesion kinase/extracellular signal-regulated kinase/matrix metalloproteinase-9 axis. Hepatology 54: 1808-1818, 2011.

25. Nieto $\mathrm{N}$ and Cederbaum AI: Increased Sp1-dependent transactivation of the LAMgamma 1 promoter in hepatic stellate cells co-cultured with HepG2 cells overexpressing cytochrome P450 2E1. J Biol Chem 278: 15360-15372, 2003.

26. Zhang HY and Sun H: Up-regulation of Foxp3 inhibits cell proliferation, migration and invasion in epithelial ovarian cancer. Cancer Lett 287: 91-97, 2010.

27. Guo LY, Li YM, Qiao L, Liu T, Du YY, Zhang JQ, He WT, Zhao YX and He DQ: Notch2 regulates matrix metallopeptidase 9 via PI3K/AKT signaling in human gastric carcinoma cell MKN-45. World J Gastroenterol 18: 7262-7270, 2012.

28. Mohla S and Witz IP: The 5th International Conference on Tumor Microenvironment: Progression, therapy and prevention. Versailles, France, October 20-24, 2009: conference summary. Cancer Microenviron 3: 1-5, 2010.

29. Mbeunkui F and Johann DJ Jr: Cancer and the tumor microenvironment: A review of an essential relationship. Cancer Chemother Pharmacol 63: 571-582, 2009.

30. Paget S: The distribution of secondary growths in cancer of the breast. 1889. Cancer Metastasis Rev 8: 98-101, 1989.

31. Qayed M and Katzenstein HM: Dose-intensive cisplatin for hepatoblastoma: Have you heard? Lancet Oncol 14: 791-792, 2013.

32. Xu N, Shen C, Luo Y, Xia L, Xue F, Xia Q and Zhang J: Upregulated miR-130a increases drug resistance by regulating RUNX3 and Wnt signaling in cisplatin-treated HCC cell. Biochem Biophys Res Commun 425: 468-472, 2012.

33. Wang H, Tan G, Dong L, Cheng L, Li K, Wang Z and Luo H: Circulating MiR-125b as a marker predicting chemoresistance in breast cancer. PLoS One 7: e34210, 2012.

34. Gatz SA and Wiesmüller L: p53 in recombination and repair. Cell Death Differ 13: 1003-1016, 2006.

35. Pandit B and Gartel AL: Proteasome inhibitors induce p53-independent apoptosis in human cancer cells. Am J Pathol 178: 355-360, 2011. 